\title{
Fault Tolerance of Parallel Manipulators with Passive Joints
}

\author{
Pål Johan From* Jan Tommy Gravdahl* \\ * Department of Engineering Cybernetics, Norwegian University of \\ Science and Technology, 7491 Trondheim, Norway. \\ e-mail:from@itk.ntnu.no
}

\begin{abstract}
A systematic analysis of the mobility of closed chain manipulators with passive joints is given. The main observation presented in this paper is that the mobility of the manipulator, considering the passive joints only, should always be zero. Further, for the manipulator to be fault tolerant, the mobility should remain zero when actuator failure occurs for an arbitrary joint. We present a simple approach to the problem of finding the smallest set of active joints for which the manipulator remains equilibrated with respect to free swinging joint failure in any joint.
\end{abstract}

\section{INTRODUCTION}

This paper discusses the effect that passive joints have on the mobility of parallel manipulators. The main motivation and also the main example used throughout the paper is joint failure. We study the ability of the mechanism to remain equilibrated when free-swinging joint failure (FSJF) occurs, see Tinós et al. [2006].

We will denote a manipulator equilibrated if it can resist a wrench in an arbitrary direction, either through kinematic constraints or through actuator torques. We obtain this if the manipulator, considering the passive joints only, has mobility equal to zero, i.e. we do not want the passive joints to allow any motion when the active joints are locked. If this property is satisfied this is the same as guaranteeing that manipulator does not have an unstable singularity, following the classification in Matone and Roth [2006].

For non-overconstrained mechanisms, i.e. there are no redundant constraints, we can apply the well known Grübler formula. The active joints can be chosen arbitrarily as long as the manipulator remains non-overconstrained and the self-motion is considered. For overconstrained mechanisms, there are many different approaches to determine mobility. In Dai et al. [2006] the mobility of the mechanism is found from the constraint space. The constraints of the system are found systematically and the redundant constraints are identified. The mobility is then found by adding the degrees of freedom represented by these redundant constraints to the Grübler formula for nonoverconstrained mechanisms. This approach illustrates well the effect of redundant constraints in the mechanism.

The mobility can also be found by the motion space as in Rico et al. [2003] and Rico et al. [2006]. The degree of freedom of the motion of the end effector is first found. Then the degree of freedom of the self-motion manifold of each chain is added. By this approach the redundant constraints are not found directly. This approach also gives valuable in-sight on where to place redundant actuators in the mechanism.
A systematic and rigorous analysis of the mobility of closed chain mechanisms based on the theory of twists is presented. The mobility is used to determine the minimum set of active joints needed for the manipulator to be equilibrated and fault tolerant. In From and Gravdahl [2008] we present several examples of how to apply the theory presented to different mechanisms.

\section{RIGID BODY MOTION}

This section gives the background of mathematical modelling of rigid body motion. For a detailed overview of the topic, the reader is referred to Murray et al. [1994] and Meng et al. [2007].

We will use the special Euclidean group $S E(3)$ to represent the configuration space of a rigid body. In addition to its group structure, $S E(3)$ is a differentiable manifold, and is what is known as a Lie group. $S E(3)$ is thus a matrix Lie group and can be written by homogeneous coordinates

$$
S E(3)=\left\{\left[\begin{array}{ll}
R & p \\
0 & 1
\end{array}\right] \mid p \in \mathbb{R}^{3}, R \in S O(3)\right\}
$$

where $S O(3)$ is the 3 -dimensional special orthogonal group. An element $g \in S E(3)$ represents a rotation and displacement of a rigid body relative to a reference configuration. The manifold structure of $S E(3)$ is given by

$$
\Phi: S O(3) \ltimes \mathbb{R}^{3} \rightarrow S E(3):(R, p) \mapsto\left[\begin{array}{cc}
R & p \\
0 & 1
\end{array}\right] .
$$

Associated with every Lie group $G$ is its Lie algebra $\mathfrak{g}$ which is defined as the tangent space of $G$ at the identity $e$ and is written as $\mathfrak{g} \triangleq T_{e} G$. A vector space $V$ is a Lie algebra if there exists a bilinear operation given by the matrix commutator $\left[v_{1}, v_{2}\right]=v_{1} v_{2}-v_{2} v_{1}$. The Lie algebra se(3) of $S E(3)$ consist of all $4 \times 4$ matrices

$$
s e(3)=\left[\begin{array}{ll}
\hat{\omega} & v \\
0 & 0
\end{array}\right]
$$

where $v \in \mathbb{R}^{3}$ and $\hat{\omega}$ is the skew-symmetric matrix representation of $\omega \in \mathbb{R}^{3}$ 


$$
\hat{\omega}=\left[\begin{array}{ccc}
0 & -\omega_{3} & \omega_{2} \\
\omega_{3} & 0 & -\omega_{1} \\
-\omega_{2} & \omega_{1} & 0
\end{array}\right] \in s o(3) .
$$

An element of $s e(3)$ can be represented by the twist coordinates $\xi=\left[v^{\top} \omega^{\top}\right]^{\top} \in \mathbb{R}^{6}$ which can be identified with the twist $\hat{\xi} \in \operatorname{se}(3)$ by the map

$$
\wedge: \mathbb{R}^{6} \rightarrow \operatorname{se}(3): \xi=\left[\begin{array}{c}
v \\
\omega
\end{array}\right] \mapsto \hat{\xi}=\left[\begin{array}{ll}
\hat{\omega} & v \\
0 & 0
\end{array}\right] \in \operatorname{se}(3) .
$$

The exponential map

$$
\exp : \operatorname{se}(3) \rightarrow S E(3): \hat{\xi} \mapsto e^{\hat{\xi}}
$$

defines a local diffeomorphism taking the zero vector of $s e(3)$ to the identity element of $S E(3)$. Physically $e^{\theta \hat{\xi}}$ $\theta \in \mathbb{R}$ corresponds to a screw motion along the axis of a fixed $\xi$. Denote by $L_{g}$ and $R_{g}$ the left and right translation map, respectively. The differential $L_{g *}$ of $L_{g}$ defines the body velocity and the differential $R_{g *}$ of $R_{g}$ defines spatial ${ }^{1}$ velocity of a rigid body. Then for a trajectory $g(t) \in S E(3), t \in(-\epsilon, \epsilon)$, the body velocity of the rigid body is given by

$$
\hat{V}^{b}=L_{g(t)^{-1} *} \cdot \dot{g}(t)=\left[\begin{array}{cc}
R^{\top} \dot{R} & R^{\top} \dot{p} \\
0 & 0
\end{array}\right]=\left[\begin{array}{cc}
\hat{\omega} & v \\
0 & 0
\end{array}\right]
$$

while the spatial velocity is given by $\hat{V}^{s}=R_{g^{-1} *} \cdot \dot{g}$. The body and spatial velocities are related by the Adjoint map

$$
V^{s}=\operatorname{Ad}_{g} V^{b}
$$

where $g=(R, p)$ and

$$
\operatorname{Ad}_{g}=\left[\begin{array}{cc}
R & \hat{p} R \\
0 & R
\end{array}\right]
$$

We will write the twist of joint $i$ as $\mathcal{G}_{i}$ and the twist system of chain $j$ as

$$
\overline{\mathcal{M}}_{j}=\left(\mathcal{G}_{1}, \mathcal{G}_{2}, \ldots, \mathcal{G}_{n}\right) .
$$

The twist system describes the motion of the end effector of the open chain (Meng et al. [2007]).

We will introduce the following notation from Dai et al. [2006] to represent sets of twists or wrenches. Braces $\{\cdot\}$ are used to indicate a set that contains unique elements while angle brackets $\langle\cdot\rangle$ are used to indicate multisets which may contain multiple entries of each element. We will use cardinality (card) to give the number of elements in $\left\langle\overline{\mathcal{M}}_{j}\right\rangle$ or $\left\{\overline{\mathcal{M}}_{j}\right\}$. For $\left\{\overline{\mathcal{M}}_{j}\right\}$, the cardinality is equal to the dimension. Let the parallel manipulator

$$
\mathcal{M}=\mathcal{M}_{1}\left\|\mathcal{M}_{2}\right\| \cdots \| \mathcal{M}_{k}
$$

consist of $k$ serial manipulator sub-chains that share a common base and a common end effector. The set of endeffector motions is defined as (Meng et al. [2007])

$$
C_{\mathcal{M}}=C_{\mathcal{M}_{1}} \cap C_{\mathcal{M}_{2}} \cap \cdots \cap C_{\mathcal{M}_{k}},
$$

where $C_{\mathcal{M}_{j}}$ is the set of rigid transformations that $\mathcal{M}_{j}$ generates without loop constraints. $C_{\mathcal{M}}$ defines the configurations of the end effector with the loop constraints imposed.

We are interested in the passive motion, i.e. the motion due to the passive joints when the active joints are fixed. We denote this by

$$
\mathcal{M}_{P}=\mathcal{M}_{P 1}\left\|\mathcal{M}_{P 2}\right\| \cdots \| \mathcal{M}_{P k}
$$

\footnotetext{
1 In this context, spatial means that the velocity is given with respect to a globally defined coordinate system. We will also use spatial for the 3-dimensional space, as opposed to the planar case.
}

where $\mathcal{M}_{P j}$ consists of only the passive joints of manipulator $j$.

Although only the passive joints are considered, the twists of the passive joints depend on the configuration of the active joints. The twist of joint $i$ is given by

$$
\mathcal{G}_{i}^{\prime}=\operatorname{Ad}_{g_{(i-1)}} \mathcal{G}_{i}
$$

where $g_{i} \in S E(3)$ is the transformation from the base to joint $i$. We will assume it implicitly understood that the twists, as written in (10), are transformed according to (14), and thus write $\mathcal{G}$ for $\mathcal{G}^{\prime}$.

We will find the mobility $D$ considering the passive joints only. If the mobility of the mechanism is zero we conclude that the mechanism is equilibrated with respect to any external force. On the other hand, if $D>0$ an additional condition needs to be satisfied for the mechanism to be equilibrated. This is not considered in this paper. We will denote a mechanism equilibrated if the following is satisfied:

Definition 2.1. A manipulator $\mathcal{M}$ is denoted equilibrated with respect to an external wrench $F_{\text {ext }}=\left[f^{\top} \tau^{\top}\right]^{\top}$ where $f, \tau \in \mathbb{R}^{3}$, if $\mathcal{M}$, either through kinematic constraints or through actuator torque, can produce a wrench opposite to $F_{\text {ext }}$, i.e. $\mathcal{M}$ can produce the wrench $-k F_{\text {ext }}$ for some $k>0$.

Note that we do not require that the manipulator is able to resist any external wrench, only that it can produce a wrench of a given type and direction.

\section{OVERCONSTRAINED MECHANISMS}

Grübler's formula gives the mobility of mechanisms with linearly dependent constraints (non-overconstrained). For the three-dimensional Euclidean space, Grübler's formula is given by

$$
D=6 N-\sum_{i=1}^{n}\left(6-f_{i}\right),
$$

where $f_{i}$ is 1 for the 1 -dimensional lower pairs and we write

$$
D=6 N-5 n \text {. }
$$

When the constraints are not linearly independent, the mechanism is over-constrained, i.e. some of the constraints are redundant and have no effect on the mobility. Based on the approach in Dai et al. [2006] we first identify the constraints that are common for all chains and eliminate the redundancy in this set. This set is easily identified as the intersection of the constraint space of all the chains. Further the constraints that constrain each chain to the end-effector motion, the end effector constraint system, are identified and again the redundant constraints are found from this set. The approach presented in Dai et al. [2006] is based on the screw system of the mechanism and represents the constraint space as reciprocal screws. Here, we apply the same general idea as in Dai et al. [2006]. The approach is based on an analysis of the sub-algebras and sub-manifolds of the Lie Algebra se(3) and their cotangent spaces.

The approach is general in the sense that no classification of the mechanism is required. As pointed out in Rico et al. [2003], the classification of the mechanism is not needed 
to determine its mobility. However, in our setting, the classification is important in the sense that it tells us where to place the active joints. As for the non-overconstrained case, the active joints cannot be placed arbitrarily in the mechanism. We will see that only in very special cases can the active joints be arbitrarily placed in the mechanism.

The approach in Dai et al. [2006] is based on the constraint space formulation. It is also shown that the mobility can be found by the motion space as in Rico et al. [2003] and Rico et al. [2006]. By this approach the chains are also classified and it is straight forward to determine the effect a passive joint has on the mechanism. We will use the motion space approach to set up a set of simple rules on where to place the active joints in the mechanism in order for the mechanism to be equilibrated. This set of rules naturally leads to an approach on how to choose actuator redundancy most efficiently to make the manipulator resistant to joint failures.

\subsection{The Constraint Space}

To find the mobility from the constraint space as in Dai et al. [2006], we start by denoting the motion space of the chain $j$ as

$$
\overline{\mathcal{M}}_{j}=\left(\mathcal{G}_{1}, \mathcal{G}_{2}, \ldots, \mathcal{G}_{n_{j}}\right)
$$

where $\mathcal{G}_{i}$ is the twist of joint $i$ and $n_{j}$ is the number of joints in chain $j$. Recall that braces $\{\cdot\}$ are used to indicate a set that contains unique elements while angle brackets $\langle\cdot\rangle$ are used to indicate multisets which may contain multiple entries of each element. If the twists of the joints in chain $j$ are linearly independent we have that $\sum_{i=1}^{n_{j}} f_{i}=\operatorname{card}\left\{\overline{\mathcal{M}}_{j}\right\}$. If this is not the case, then $\sum_{i=1}^{n_{j}} f_{i}=\operatorname{card}\left\langle\overline{\mathcal{M}}_{j}\right\rangle>\operatorname{card}\left\{\overline{\mathcal{M}}_{j}\right\}$ and the chain is redundant in itself, i.e. it may have self-motion. In this case singularities may also occur in the chain. This will not be considered further.

We will denote the constraint system of chain $j$ as

$$
\overline{\mathcal{M}}_{j}^{C}=\overline{\mathcal{M}}_{j}^{\perp}
$$

where $\overline{\mathcal{M}}_{j}^{\perp}=\left\{F \in \mathbb{R}^{6} \mid F \cdot V=0, \forall V \in \overline{\mathcal{M}}_{j}\right\}$ which is the vanishing of the reciprocal product of Ball (Lipkin and Duffy [2002]). This represents the constraints imposed on the end effector by chain $j$. Note that we cannot identify the self-motion from $\overline{\mathcal{M}}_{j}^{C}$.

Further we will define mechanism motion as the union of all the twists in the system

$$
\overline{\mathcal{M}}_{M}=\overline{\mathcal{M}}_{1} \cup \overline{\mathcal{M}}_{2} \cup \cdots \cup \overline{\mathcal{M}}_{k},
$$

evaluated at $g \in C_{\mathcal{M}_{1}} \cap \cdots \cap C_{\mathcal{M}_{k}}$. The end-effector constraints is given as the union of the constraints of each chain,

$$
\overline{\mathcal{M}}_{E}^{C}=\overline{\mathcal{M}}_{1}^{C} \cup \overline{\mathcal{M}}_{2}^{C} \cup \cdots \cup \overline{\mathcal{M}}_{k}^{C}
$$

evaluated at $g \in C_{\mathcal{M}_{1}} \cap \cdots \cap C_{\mathcal{M}_{k}}$. From this we can find the constrained motion of the end effector

$$
\overline{\mathcal{M}}_{E}=\left(\overline{\mathcal{M}}_{E}^{C}\right)^{\perp} \text {. }
$$

The intersection of all the constraints are further given by or alternatively

$$
\overline{\mathcal{M}}_{M}^{C}=\overline{\mathcal{M}}_{1}^{C} \cap \overline{\mathcal{M}}_{2}^{C} \cap \cdots \cap \overline{\mathcal{M}}_{k}^{C},
$$

$$
\overline{\mathcal{M}}_{M}^{C}=\left(\overline{\mathcal{M}}_{M}\right)^{\perp}
$$

With the notation of braces and angle brackets, each of the subsets introduced in this section is given by

$$
\begin{aligned}
\left\{\overline{\mathcal{M}}_{M}\right\} & =\bigcup_{j=1}^{k} \overline{\mathcal{M}}_{j}, & \left\langle\overline{\mathcal{M}}_{M}\right\rangle & =\sum_{j=1}^{k} \overline{\mathcal{M}}_{j}, \\
\left\{\overline{\mathcal{M}}_{E}^{C}\right\} & =\bigcup_{j=1}^{k} \overline{\mathcal{M}}_{j}^{\perp}, & \left\langle\overline{\mathcal{M}}_{E}^{C}\right\rangle & =\sum_{j=1}^{k} \overline{\mathcal{M}}_{j}^{\perp}, \\
\left\{\overline{\mathcal{M}}_{E}\right\} & =\bigcap_{j=1}^{k} \overline{\mathcal{M}}_{j}, & \left\{\overline{\mathcal{M}}_{M}^{C}\right\} & =\sum_{j=1}^{k} \overline{\mathcal{M}}_{j}^{C}, \\
\left\{\overline{\mathcal{M}}_{E}\right\} & =\left\{\overline{\mathcal{M}}_{E}^{C}\right\}^{\perp} & \left\{\overline{\mathcal{M}}_{M}^{C}\right\} & =\left\{\overline{\mathcal{M}}_{M}\right\}^{\perp} \\
& =\left\langle\overline{\mathcal{M}}_{E}^{C}\right\rangle^{\perp}, & & =\left\langle\overline{\mathcal{M}}_{M}\right\rangle^{\perp} .
\end{aligned}
$$

Thus, the collection of all constraints is given in $\left\langle\overline{\mathcal{M}}_{E}^{C}\right\rangle$, including repeated elements. The first step is to factorise out all the constraints that are common for all chains. The "directions" of the end effector represented by these constraints can be considered the most robust directions as they are constrained by all the sub-chains in the mechanism. The constraints that are common for all subchains is given by $\left\{\overline{\mathcal{M}}_{M}^{C}\right\}$. We will say that a single subchain cannot have redundant constraints (as seen from the other chains or the end effector). This is always true. Because $\left\{\overline{\mathcal{M}}_{M}^{C}\right\}$ is the same for all chains we will write

$$
\left\langle\overline{\mathcal{M}}_{M}^{C}\right\rangle=k \cdot\left\{\overline{\mathcal{M}}_{M}^{C}\right\} .
$$

We see that $\left\langle\overline{\mathcal{M}}_{M}^{C}\right\rangle$ is $(k-1)$ times redundant.

For each chain we can factorise out this part by taking

$$
\left\{\overline{\mathcal{M}}_{j}^{C}\right\}=\left\{\overline{\mathcal{M}}_{M}^{C}\right\} \cup\left\{\overline{\mathcal{M}}_{C j}^{C}\right\}
$$

We can add the multisets of Equation (25) and get

$$
\begin{aligned}
\left\langle\overline{\mathcal{M}}_{E}^{C}\right\rangle & =\left\langle\overline{\mathcal{M}}_{M}^{C}\right\rangle+\left\langle\overline{\mathcal{M}}_{C}^{C}\right\rangle \\
& =k \cdot\left\{\overline{\mathcal{M}}_{M}^{C}\right\}+\left\langle\overline{\mathcal{M}}_{C}^{C}\right\rangle .
\end{aligned}
$$

As the redundancy in $\left\langle\overline{\mathcal{M}}_{M}^{C}\right\rangle$ is already dealt with, we can focus on $\left\langle\overline{\mathcal{M}}_{C}^{C}\right\rangle$ which may also be redundant. We start by writing

$$
\left\langle\overline{\mathcal{M}}_{C}^{C}\right\rangle=\left\{\overline{\mathcal{M}}_{C}^{C}\right\}+\left\langle\overline{\mathcal{M}}_{\nu}^{C}\right\rangle .
$$

Here, $\left\{\overline{\mathcal{M}}_{C}^{C}\right\}$ is the linearly independent part which restricts the motion of the end effector to $\overline{\mathcal{M}}_{E}$, while $\left\langle\overline{\mathcal{M}}_{\nu}^{C}\right\rangle$ is the collection of the constraints that are linearly dependent of the entries in $\left\{\overline{\mathcal{M}}_{C}^{C}\right\}$. Thus, the redundancy given by the term $\left\langle\overline{\mathcal{M}}_{C}^{C}\right\rangle$ in Equation (26) is given by $\operatorname{card}\left\langle\overline{\mathcal{M}}_{\nu}^{C}\right\rangle$. The total redundancy of the system is given by

$$
\operatorname{card}\left\langle\overline{\mathcal{M}}_{E \nu}^{C}\right\rangle=(k-1) \operatorname{card}\left\{\overline{\mathcal{M}}_{M}^{C}\right\}+\operatorname{card}\left\langle\overline{\mathcal{M}}_{\nu}^{C}\right\rangle .
$$

Finally, we also note that $\left\langle\overline{\mathcal{M}}_{E \nu}^{C}\right\rangle$ can also be factorised out from

$$
\left\langle\overline{\mathcal{M}}_{E}^{C}\right\rangle=\left\{\overline{\mathcal{M}}_{E}^{C}\right\}+\left\langle\overline{\mathcal{M}}_{E \nu}^{C}\right\rangle .
$$

\subsection{The Modified Grübler Formula}

The Grübler formula does not take redundant constraints into consideration. Redundant constraint are constraints that do not reduce the mobility of the end effector or the chains. We therefore need to add these to the Grübler 
formula. The Modified Grübler formula as presented in Dai et al. [2006] is given by adding (28) to (15)

$D=d N-\sum_{i=1}^{n}\left(d-f_{i}\right)+(k-1) \cdot \operatorname{card}\left\{\overline{\mathcal{M}}_{M}^{C}\right\}+\operatorname{card}\left\langle\overline{\mathcal{M}}_{\nu}^{C}\right\rangle$

where $d$ is the dimension of the space, normally 3 or 6 . This expression identifies the redundant constraints. It also gives the mobility due to self-motion. It does not, however, identify very easily due to what joints these motions occur. This is considered in more detail in the next section.

\subsection{The Motion Space}

The mobility of the mechanism tells us how many active joints are needed for the mechanism to be equilibrated. However, it does not tell us what joints can be set as passive and what joints need to be active. In the following, we will show that an alternative to the Modified Grübler formula given in (30) can be found from the motion space and we will show how this approach naturally leads to the classification of different types of overconstrained joints. This is the same classification of overconstrained chains as in Rico et al. [2003] and Rico et al. [2006]. Further, in the next section, we will use this to set up a set of simple rules on where the active joints need to be placed, i.e. how many active joints need to be placed in each chain, as well as their position in the chain.

Exceptional Linkages In Rico et al. [2003], two subchains that have an intersection, but for which the motion space of one sub-chain is not a subspace of the other, is denoted exceptional linkage. We refer to the work of Hervé [1978] for a formal definition of exceptional, trivial and paradoxical linkages. Paradoxical linkages are not treated here. In Rico et al. [2006] this is generalised to the case of arbitrarily many chains. Here we will look at it from a different view in order to get a deeper understanding of the mobility criteria.

We start by finding the mobility of the end effector. This is given by the intersection of the motion space of each chain

$$
\left\{\overline{\mathcal{M}}_{E}\right\}=\bigcap_{j=1}^{k}\left\{\overline{\mathcal{M}}_{j}\right\}
$$

evaluated at $g \in C_{\mathcal{M}_{1}} \cap \cdots \cap C_{\mathcal{M}_{k}}$. Thus the mobility of the end effector is given by $\operatorname{card}\left\{\overline{\mathcal{M}}_{E}\right\}$. Each chain may also have a mobility independent of the mobility of the end effector. This is the self-motion and is given by the total number of freedoms in the chain and substracting the dimension of the open loop end-effector motion of the chain.

$$
\begin{aligned}
\operatorname{card}\left\{\overline{\mathcal{M}}_{s m}\right\}_{j} & =\sum_{i=1}^{n_{j}} f_{i}-\operatorname{card}\left\{\overline{\mathcal{M}}_{j}\right\} \\
\operatorname{card}\left\{\overline{\mathcal{M}}_{s m}\right\} & =\sum_{j=1}^{k}\left(\sum_{i=1}^{n_{j}} f_{i}-\operatorname{card}\left\{\overline{\mathcal{M}}_{j}\right\}\right) .
\end{aligned}
$$

The total mobility of the mechanism is then given by adding (32) to (31). We will write this as a proposition and provide a different proof than that of Rico et al. [2006].
Proposition 1. The total mobility of a mechanism is given by the degree of freedom of the end effector, given in Equation (31) and the self-motion of the chain, given in Equation (32) by

$$
\begin{aligned}
D & =\operatorname{card}\left\{\overline{\mathcal{M}}_{E}\right\}+\sum_{j=1}^{k}\left(\sum_{i=1}^{n_{j}} f_{i}-\operatorname{card}\left\{\overline{\mathcal{M}}_{j}\right\}\right) \\
& =\operatorname{card}\left\{\overline{\mathcal{M}}_{E}\right\}+\sum_{i=1}^{n} f_{i}-\sum_{j=1}^{k} \operatorname{card}\left\{\overline{\mathcal{M}}_{j}\right\}
\end{aligned}
$$

Proof 1. (sketch) We need to show that a) the degree of freedom of the end effector is given by the dimension of $\left\{\overline{\mathcal{M}}_{E}\right\}$ in Equation (31); and b) that the self-motion of each chain is given by Equation (32).

a) The degree of freedom of the end effector is given by the dimension of $\left\{\overline{\mathcal{M}}_{E}\right\}$ in Equation (31). This follows directly from Meng et al. [2007].

b) The dimension of the self-motion manifold can be found in most textbooks on robotics (e.g. Murray et al. [1994]) to be the dimension of the null of the Jacobian $\mathcal{N}(J)$, which is given as $\operatorname{dim}(\mathcal{N}(J))=n_{j}-$ $m$ where $n_{j}$ is the number of joints and $m$ is the dimension of the end-effector motion. This equivalent to $(32)$.

We are mainly concerned with the effect of adding a joint to the chain. Adding a joint to a chain $\mathcal{M}$ will have the same effect as making one joint passive when only the passive joints of the manipulator are considered, i.e. adding a joint to $\mathcal{M}_{P}$. In this section we look at the effect of adding a joint to the mechanisms and in the next section we use these results to analyse in what case the manipulator is equilibrated. We will use the reasoning in Proposition 1 and the observation that a joint that increases the dimension of the end effector motion of the open chain, but not of the closed chain, will always be locked. Then there are three different outcomes of adding a joint to the manipulator:

- $\sum_{i=1}^{n_{j}} f_{i}$ increases by one while $\operatorname{card}\left\{\overline{\mathcal{M}}_{j}\right\}$ does not.

- The dimension of the self-motion manifold of the chain increases by one.

- $\sum_{i=1}^{n_{j}} f_{i}$ and card $\left\{\overline{\mathcal{M}}_{j}\right\}$ increase by one while $\operatorname{card}\left\{\overline{\mathcal{M}}_{E}\right\}$ does not.

- The joint will be locked and the mobility of the system does not change.

- $\sum_{i=1}^{n_{j}} f_{i}, \operatorname{card}\left\{\overline{\mathcal{M}}_{j}\right\}$ and card $\left\{\overline{\mathcal{M}}_{E}\right\}$ increase by one.

- The mobility of the end effector increases by one.

- The dimension of the self-motion manifold of the chain does not increase.

Thus, by checking the dimension of $\operatorname{card}\left\{\overline{\mathcal{M}}_{j}\right\}$ and $\operatorname{card}\left\{\overline{\mathcal{M}}_{E}\right\}$ we can effectively find the effect that a joint failure has on the mobility of the mechanism.

Trivial Linkage of Type I In Rico et al. [2006] the case when all the sub-chains generate the same motion

$$
\left\{\overline{\mathcal{M}}_{j}\right\}=\left\{\overline{\mathcal{M}}_{E}\right\} \quad \text { for } j=1 \ldots k
$$

is denoted trivial linkages. In this case the mobility is found directly from (33) by 


$$
\begin{aligned}
D & =\operatorname{card}\left\{\overline{\mathcal{M}}_{E}\right\}+\sum_{j=1}^{k}\left(\sum_{i=1}^{n_{j}} f_{i}-\operatorname{card}\left\{\overline{\mathcal{M}}_{E}\right\}\right) \\
& =\operatorname{card}\left\{\overline{\mathcal{M}}_{E}\right\}-k \cdot \operatorname{card}\left\{\overline{\mathcal{M}}_{E}\right\}+\sum_{j=1}^{k}\left(\sum_{i=1}^{n_{j}} f_{i}\right) \\
& =\sum_{i=1}^{n} f_{i}-(k-1) \cdot \operatorname{card}\left\{\overline{\mathcal{M}}_{E}\right\} .
\end{aligned}
$$

In this case there are only two different outcomes:

- $\sum_{i=1}^{n_{j}} f_{i}$ increases by one while $\operatorname{card}\left\{\overline{\mathcal{M}}_{j}\right\}$ does not.

- The dimension of the self-motion manifold of the chain increases by one.

- $\sum_{i=1}^{n_{j}} f_{i}$ and card $\left\{\overline{\mathcal{M}}_{j}\right\}$ increase by one.

- The joint will be locked and the mobility of the system does not change.

- $\sum_{i=1}^{n_{j}} f_{i}, \operatorname{card}\left\{\overline{\mathcal{M}}_{j}\right\}$ and card $\left\{\overline{\mathcal{M}}_{E}\right\}$ increase by one. - Impossible

Trivial Linkage of Type II The case when the entire motion of the end-effector, connected by two sub-chains, can be determined and is restricted by one chain is denoted trivial linkage. As they only consider single loops, the constraints of the other chain does not affect the mobility of the end effector. In our setting, we define the corresponding multi-loop classification of trivial linkage as the following. Assume that we have $E^{\prime}$ manipulators that all generate $\overline{\mathcal{M}}_{E}$ and $M^{\prime}$ manipulators that all generate $\overline{\mathcal{M}}_{M}$, where $\overline{\mathcal{M}}_{E} \subset \overline{\mathcal{M}}_{M}$. Then the total mobility of the system is given by $\overline{\mathcal{M}}_{E}$ and the internal mobility of each of the chains. The internal mobility of the chains in $\mathcal{M}_{E}$ and $\mathcal{M}_{M}$ must, however, be treated differently. This can be generalised to $\overline{\mathcal{M}}_{E_{1}} \subset \overline{\mathcal{M}}_{E_{2}} \subset \cdots \subset \overline{\mathcal{M}}_{M}$.

The total mobility of the system is then given by

$$
\begin{aligned}
D= & \operatorname{card}\left\{\overline{\mathcal{M}}_{E}\right\}+\sum_{j=1}^{k}\left(\sum_{i=1}^{n_{j}} f_{i}-\operatorname{card}\left\{\overline{\mathcal{M}}_{j}\right\}\right) \\
= & \operatorname{card}\left\{\overline{\mathcal{M}}_{E}\right\}+\sum_{E}\left(\sum_{i=1}^{n_{j}} f_{i}-\operatorname{card}\left\{\overline{\mathcal{M}}_{E}\right\}\right) \\
& +\sum_{M}\left(\sum_{i=1}^{n_{j}} f_{i}-\operatorname{card}\left\{\overline{\mathcal{M}}_{M}\right\}\right) \\
= & \sum_{i=1}^{n} f_{i}-\left(E^{\prime}-1\right) \cdot \operatorname{card}\left\{\overline{\mathcal{M}}_{E}\right\}-M^{\prime} \cdot \operatorname{card}\left\{\overline{\mathcal{M}}_{M}\right\}
\end{aligned}
$$

where $\sum_{E}$ sums over all the chains that generate $\overline{\mathcal{M}}_{E}$ and $\sum_{M}$ sums over all the chains that generate $\overline{\mathcal{M}}_{M}$. Also in this case there are three different outcomes which we will divide into two classes:

For $j \in M$,

- $\sum_{i=1}^{n_{j}} f_{i}$ increases by one while $\operatorname{card}\left\{\overline{\mathcal{M}}_{j}\right\}$ does not.

- The dimension of the self-motion manifold of the chain increases by one.

- $\sum_{i=1}^{n_{j}} f_{i}$ and $\operatorname{card}\left\{\overline{\mathcal{M}}_{j}\right\}$ increase by one while $\operatorname{card}\left\{\overline{\mathcal{M}}_{E}\right\}$ does not.

- The joint will be locked and the mobility of the system does not change.

- $\sum_{i=1}^{n_{j}} f_{i}, \operatorname{card}\left\{\overline{\mathcal{M}}_{M}\right\}$ and $\operatorname{card}\left\{\overline{\mathcal{M}}_{E}\right\}$ increase by one.

- Impossible. $j \in E$,

- $\sum_{i=1}^{n_{j}} f_{i}$ increases by one while $\operatorname{card}\left\{\overline{\mathcal{M}}_{j}\right\}$ does not.

- The dimension of the self-motion manifold of the chain increases by one.

- $\sum_{i=1}^{n_{j}} f_{i}$ and $\operatorname{card}\left\{\overline{\mathcal{M}}_{j}\right\}$ increase by one while $\operatorname{card}\left\{\overline{\mathcal{M}}_{E}\right\}$ does not.

- The joint will be locked and the mobility of the system does not change.

- $\sum_{i=1}^{n_{j}} f_{i}, \operatorname{card}\left\{\overline{\mathcal{M}}_{M}\right\}$ and $\operatorname{card}\left\{\overline{\mathcal{M}}_{E}\right\}$ increase by one.

- The mobility of the end effector increases by one.

- The self-motion of the chain does not increase.

\section{FAULT TOLERANCE}

In this section, we look into the effect of FSJF in parallel manipulators and how the results from the previous section can be used to prevent that the mechanism turns inequilibrated. For a general treatment and an approach on how to identify joint failure see Tinós et al. [2006]. In this case, as the number of passive joints in the manipulator increases by one, the mobility of $\mathcal{M}_{P}$ may remain zero or increase by one. Let $m$ be the number of active joints in $\mathcal{M}$. When $\mathcal{M}_{P}$ does not allow any motion after the joint failure, we have

$$
D_{m}=0 \stackrel{F S J F}{\rightleftharpoons} D_{m-1}=0
$$

and the manipulator remains equilibrated with respect to external forces. When $\mathcal{M}_{P}$ allows a 1 DOF motion as a result of the joint failure, i.e.

$$
D_{m}=0 \stackrel{\text { FSJF }}{\rightleftharpoons} D_{m-1}=1,
$$

the mechanism is not fault tolerant.

We are interested in the condition for which $D_{m-1}=0$. As seen in the previous section, the effect of a free-swinging joint failure depends on the joint. We start by setting up a set of rules that determines if a joint failure will increase the mobility of $\mathcal{M}_{P}$. This can also be used as a design criterion to guarantee the mechanism to be fault tolerant. We do that by determining where to put the actuator redundancy most effectively in order for the manipulator to be resistant to a joint failure of any joint.

In the following we will find the conditions for which the mechanism is equilibrated for all the different outcomes of joint failure found in Section 3.3.

\section{Exceptional Linkages}

- $\sum_{i=1}^{n_{j}} f_{i}$ increases by one while $\operatorname{card}\left\{\overline{\mathcal{M}}_{j}\right\}$ does not.

- The end effector is equilibrated. Chain $j$ will only remain equilibrated if it is actuator redundant. The redundancy must be in the set of joints in which the self-motion occurs.

- $\sum_{i=1}^{n_{j}} f_{i}$ and $\operatorname{card}\left\{\overline{\mathcal{M}}_{j}\right\}$ increase by one while $\operatorname{card}\left\{\overline{\mathcal{M}}_{E}\right\}$ does not.

- No action needed. Both end effector and chains are equilibrated.

- $\sum_{i=1}^{n_{j}} f_{i}, \operatorname{card}\left\{\overline{\mathcal{M}}_{j}\right\}$ and $\operatorname{card}\left\{\overline{\mathcal{M}}_{E}\right\}$ increase by one.

- The mobility can be compensated with actuator redundancy in any joint that is not locked for the motion generated by $\mathcal{M}_{P}$.

The last case illustrates an important difference between the overconstrained and non-overconstrained case. For 
the non-overconstrained case the active joints can be placed arbitrarily in the mechanisms while for the nonoverconstrained case, the active joints must be chosen so that they generate the end-effector motion. In the nonoverconstrained case this is taken care of by applying the planar and spatial Grübler formula to the mechanism. For the overconstrained case, the motion of the end effector must be considered.

\section{Trivial Linkage of Type I}

- $\sum_{i=1}^{n_{j}} f_{i}$ increases by one while card $\left\{\overline{\mathcal{M}}_{j}\right\}$ does not.

- The end effector is equilibrated. Chain $j$ will only remain equilibrated if it is actuator redundant. The redundancy must be in the set of joints in which the self-motion occurs.

- $\sum_{i=1}^{n_{j}} f_{i}$ and card $\left\{\overline{\mathcal{M}}_{j}\right\}$ increase by one.

- No action needed. Both end effector and chains are equilibrated.

- $\sum_{i=1}^{n_{j}} f_{i}, \operatorname{card}\left\{\overline{\mathcal{M}}_{j}\right\}$ and $\operatorname{card}\left\{\overline{\mathcal{M}}_{E}\right\}$ increase by one.

- Will never occur.

Trivial Linkage of Type II

For $j \in M$,

- $\sum_{i=1}^{n_{j}} f_{i}$ increases by one while $\operatorname{card}\left\{\overline{\mathcal{M}}_{j}\right\}$ does not.

- The end effector is equilibrated. Chain $j$ will only remain equilibrated if it is actuator redundant. The redundancy must be in the set of joints in which the self-motion occurs.

- $\sum_{i=1}^{n_{j}} f_{i}$ and card $\left\{\overline{\mathcal{M}}_{j}\right\}$ increase by one while $\operatorname{card}\left\{\overline{\mathcal{M}}_{E}\right\}$ does not.

- No action needed. Both end effector and chains are equilibrated.

- $\sum_{i=1}^{n_{j}} f_{i}, \operatorname{card}\left\{\overline{\mathcal{M}}_{M}\right\}$ and $\operatorname{card}\left\{\overline{\mathcal{M}}_{E}\right\}$ increase by one.

- Will never occur.

For $j \in E$,

- $\sum_{i=1}^{n_{j}} f_{i}$ increases by one while card $\left\{\overline{\mathcal{M}}_{j}\right\}$ does not.

- The end effector is equilibrated. Chain $j$ will only remain equilibrated if it is actuator redundant. The redundancy must be in the set of joints in which the self-motion occurs.

- $\sum_{i=1}^{n_{j}} f_{i}$ and card $\left\{\overline{\mathcal{M}}_{j}\right\}$ increase by one while card $\left\{\overline{\mathcal{M}}_{E}\right\}$ does not.

- No action needed. Both end effector and chains are equilibrated.

- $\sum_{i=1}^{n_{j}} f_{i}, \operatorname{card}\left\{\overline{\mathcal{M}}_{M}\right\}$ and $\operatorname{card}\left\{\overline{\mathcal{M}}_{E}\right\}$ increase by one.

- The mobility can be compensated with actuator redundancy in any joint that is not locked for the motion generated by $\mathcal{M}_{P}$.

From the results presented in this section, we see that we can easily verify if redundant actuation is needed when joint failure occurs for a given active joint. If joint failure does not lead to self-motion it may be compensated for by another redundant actuated joint that is not locked for this motion. These cases are important to recognise in order to not place unnecessary many active joints in the mechanism. If, on the other hand, the joint failure leads to self-motion, a redundant actuated joint is always needed in the respective chain. These observations lead to a simple rule on how to place the redundant active joints to guarantee that the manipulator remains equilibrated when actuator failure occurs for an arbitrary joint.

\section{CONCLUSION}

A set of rules on how to place redundant actuators in parallel mechanisms to guarantee that the manipulator remains equilibrated when actuator failure occurs is presented. The manipulator is said to be equilibrated when the manipulator, considering the passive joints only, has no mobility. Actuator failure can be divided into three main classes. The first is when no redundant actuation is needed as the joint for which the actuator failure occurs will be locked. The second case is when the actuator failure occurs in a set of joint which generates an internal motion of a sub-chain. In this case actuator redundancy must be placed in this set of joints. When the joint does not generate an internal motion and is not locked, actuator failure can be compensated by redundancy in any part of the mechanism which is not locked, including a joint that generates internal motion. By identifying the type of the linkage we can eliminate some of the cases above.

\section{ACKNOWLEDGEMENTS}

The authors wish to acknowledge the support of the Norwegian Research Council and the TAIL IO project for their continued funding and support for this research. The TAIL IO project is an international cooperative research project led by StatoilHydro and an R\&D consortium consisting of ABB, IBM, Aker Solutions and SKF. During the work with this paper the first author was with the Hong Kong University of Science and Technology and University of California at Berkeley.

\section{REFERENCES}

R. Tinós, M. H. Terra and M. Bergerman, A fault tolerance framework for cooperative robotic manipulator Control Engineering Practice, Vol. 15, 2006.

R. Matone and B. Roth, In-Parallel Manipulators: A Framework on How to Model Actuation Schemes and a Study of their Effects on Singular Postures Transactions of ASME, Vol. 121, 1999.

J. S. Dai and Z. H. and H. Lipkin, Mobility of overconstrained parallel mechanisms Transactions of ASME, Vol. 128, 2006.

J.M. Rico, J Gallardo and B Ravani, Lie algebra and the mobility of kinematic chains Journal of Robotic Systems, Vol. 20, No. 8, 2003.

J. M. Rico, L. D. Aguilera, J. Gallardo and R. Rodriguez and H. Orozco and J. M. Barrera, A more general mobility criterion for parallel mechanisms Journal of Mechanical Design, Vol. 128, 2006.

P. J. From and J. T. Gravdahl, On the Mobility and Fault Tolerance of Closed Chain Manipulators with Passive Joints Modeling, Identification and Control, Vol 29, no 4, 2008.

R. M. Murray, Z. Li and S. Sastry, A mathematical introduction to robotic manipulation CRC Press, 1994.

J. Meng, G. Liu and Z. Li, A Geometric Theory for Analysis and Synthesis of Sub-6 DoF Parallel Manipulators IEEE Transactions on robotics, Vol. 23, No. 4, 2007.

H. Lipkin and J. Duffy, Sir Robert Stawell Ball and methodologies of modern screw theory Journal of Mechanical Engineering Science, Vol. 216, No. 1, 2002.

J. M. Hervé, Analyse structurelle des mécanismes par groupe des déplacements Mechanism Machine Theory, Vol. 13, No 4, 1978. 